\title{
Personality Based On Colour Preferences and Its Relationship with
}

\section{Achievement Motivation}

\author{
Athilakshmi. $\mathrm{R}^{1 *}$, Cynthia Deb ${ }^{2}$, Maharishi ${ }^{1}$, Ganesh Kumar ${ }^{1}$, \\ Maya Ratnasabapathy ${ }^{3}$
}

\section{ABSTRACT}

The primary objective of this investigation was to find out if there existed any relationship between the personality traits of people classified on the basis of their colour preferences and their respective achievement motivation. The study sample consisted of 110 college students (57 males and 53 females) randomly selected from diverse backgrounds within the age group of 1822 years. The research instruments used were "True Colours Personality Test” by Don Lowry to measure the difference in personality traits and the 14 item short form of Ray Achievement Motivation Scale by J. J. Ray to measure the level of Achievement Motivation. The subjects filled both the questionnaires which were then evaluated and analysed by using SPSS. It was found that subjects classified in the Green category with cool, calm, collective and logical outlook have significantly positive correlate with levels of Achievement Motivation and the Orange and Blue colour category possessing characteristics like enthusiasm, spirituality, effective communication skills, imaginative, peace loving and flexibility had a impact but not in a significant level on their Achievement Motivation, whereas those classified in Gold have a negative Pearson's Correlation coefficient indicating that the two parameters considered are inversely related. This could be attributed It is to the traits like lack of order, Dependable, Sensible and Anxious. The results and conclusions drawn from this investigation suggest that it can be applicable for a wider spectrum of the population.

Keywords: Personality, Colour and Colour Preferences, Colour and Personality, Achievement Motivation.

A plethora of studies are available for understanding how we can group personality types in various categories of colours but there is very little data on how this can be interrelated with the Achievement Motivation. The aim of this survey is to find if there is any significance between these parameters.

\footnotetext{
${ }^{1}$ Ph.D. Research Scholar, (Psychology) VIT-University, Chennai-Tamil Nadu, India

${ }^{2}$ Under Graduate Student VIT-University, Chennai-Tamil Nadu, India

${ }^{3}$ Assistant Professor Senior, (Psychology) VIT-University, Chennai-Tamil Nadu, India *Corresponding Author

(C) 2015 I Athilakshmi, C Deb, Maharishi, G Kumar, R Maya; licensee IJIP. This is an Open Access Research distributed under the terms of the Creative Commons Attribution License (http://creativecommons.org/licenses/by/2.0), which permits unrestricted use, distribution, and reproduction in any Medium, provided the original work is properly cited.
} 
Personality: The word 'personality' has been derived from the Latin root 'personna' that literally meant mask. Personality is individualistic based on a person's behaviour patterns, cognition, emotions, perception and acquired values as well as life experiences. The term has been defined by different psychologists in different ways.

According to Morton Prince personality is "Sum total of all, the biological innate dispositions, impulses, tendencies, appetites and instincts of the individual and the acquired disposition and tendencies."

"Personality is the more or less stable and enduring organization of a person's character temperament, interact and physique which determines his unique adjustment to the environment, as defined by Eysenck.

Colour and Colour Preferences: According to The Oxford Dictionary colour is the property possessed by an object of producing different sensations on the eye as a result of the way it reflects or emits light. It is a sensation of sight caused by electromagnetic waves hitting the retina.

On the other hand colour preference of an individual is related to his/her tendency to prefer some colours over the others. A person's preference for a specific colour depends upon how he/she feels in any situation, how they want to feel, and even how they remember certain experiences. Colour has long been used to create feelings of coziness or spaciousness. However, how people are affected by different colour stimuli varies from person to person.

Colour and Personality: The colour is an important aspect that builds an ambient environment for us and determines how we react and respond to the various components in our environment. According to Luscher, who advocates the thesis that the subjects with similar colour preference may have similar personality traits and the subjects' psychological reactions/attitudes to the primary colours (blue, red, yellow and green) reflect their basic psychological needs. The previous research on the dependency of personality and colour has been partially reviewed by Schaie, who suggested that the colour shades in the spectrum order from red to violet represent a series of emotions, from excitement, unrestraint, direct and prolonged impulsive discharge (red), over calmness (green), to anxiety and concern (violet). Birren has reported an association between the warm colours corresponding to energetic and excited moods and between the cool colours corresponding to passive and calm moods.

- The people classified as Orange have predominant characteristics of being Witty, Charming, Spontaneous, Impulsive, Generous, Impactful, Optimistic, Eager, Bold, Immediate and Courageous.

- Those grouped under Green are Analytical, Global, Conceptual, Cool, Calm, Collected, Inventive, Logical, Problem Solver, Abstract, Creative and Imaginative. 
- The individuals under the category Blue are Enthusiastic, Sympathetic, Personal, Warm, Communicative, Compassionate, Idealistic, Spiritual, Sincere, Peaceful, Flexible and Imaginative.

- The candidates belonging to Gold possess traits of being Loyal, Dependable, Prepared, Thorough, Sensible, Punctual, Faithful, Stable, Organized, Caring, Concerned and Helper.

Achievement Motivation: Motivation can be stated as the reasons for people's actions, desires, and needs. It can also be explained as one's direction to behaviour or what causes a person to want to repeat behaviour and vice versa. A motive is what prompts the action of a person in a certain way or at least develops an inclination for that specific action. According to Maehr and Meyer, "Motivation is a word that is part of the popular culture as few other psychological concepts are".

An achievement motive is an impulse to fulfil challenges and set a new standard of excellence that enables the individual to thrive and reach higher goals and needs. Both personality and situational factors influence achievement motivation. High-achievement motivation tends to lead to particular personality features such as persistence, ability to delay gratification, and competitiveness. Some situational factors also affect achievement motivation, including the expectation of success, incentives, control, and opportunity: Goals are most likely to increase motivation to achieve but they must be specific, challenging, achievable, and positive.

\section{REVIEW OF LITERATURE}

A number of investigations have been conducted on the colour preferences based personality categorisation and achievement motivation separately. Yet a minimal research exists that connect or link these two parameters, especially in the corporate sector.

Dunja Cigić, Vojislava Bugarski conducted the study on Personality traits and colour preferences. The investigation was to find out if there existed any differences in personality traits of subjects grouped according to the colour preference criterion. In that study they found that there were personality differences between subjects preferring dark colours (brown, grey and black) and subjects preferring bright colours (red, yellow). Social introversion and neuroticism/ anxiety were more prominent personality traits in subjects who preferred dark colours, while aggression and activity were more prominent in subjects who preferred bright colours.

Divya Ghorawat, Ravina Madan conducted a study to find out whether the colour shade preferences of individuals grouped according to their personality types are alike or not. The researchers concluded that there was no significant correlation found between the personality types and colour shade preferences of the subjects except in the case of extroverted males.

Color and Personality- Strong's Interest Inventory and Cattell's 16PF (Rense Lange, Jason Rentfrow): The research was intended to identify links between people's color preferences and 
their personal characteristics as derived from two well- established psychological inventories. The findings clearly indicate that people's color preferences as assessed through the Dewey Color System Test do provide meaningful information about their personalities, interpersonal styles, and behaviours. In particular, the data indicated that this test predicted with considerable precision all six of the Basic Interest Scales of Strong's Interest Inven- tory (Harmon, et al., 1994), and nearly all of the sixteen Primary Factors of Cattell's 16PF (Russel \& Karol, 2002).

Ekta Sharma conducted a study to show that the achievement motivation is a function of the Big five traits. As per the five factors model of personality people with high conscientiousness are considered as an organized, focused and timely achiever of their goals. So, the result is that conscientiousness is significantly related to achievement motivation.

A Quick Measure of Achievement Motivation (JJ Ray, Australian Psychologist, Vol. 14, No. 3, November 1979): This research paper was the primary information source and test tool used in this journal. A shorter version of the Ray Achievement Motivation Scale was developed and when tested on seven samples from Sydney, Glasgow, London and Johannesburg it showed reliability over 0.70 . General populations' norms as obtained from afore given four countries revealed that the English, Scots and Australians have almost same level of AM whereas South Africans have significantly higher level of AM.

\section{METHODOLOGY}

The sample was tested for to study the relationship between colour based personality and achievement motivation. The study was conducted through a questionnaire with two sections and the instructions were given prior to filling the questionnaire. It was conducted anonymously without mentioning the objective of the survey.

Sample: The survey and investigation was carried out on a sample of 110 college students belonging to an average age group of 18-22 with 57 male (54\%) and 53 female (46\%) candidates.

Tools/Instruments Used: The study was done by the application of two investigation instruments- TRUE COLOUR PERSONALITY TEST by Don Lowry and a 14 item short form of RAY ACHIEVEMENT MOTIVATION SCALE by J. J. Ray.

True Colour Personality Test: This personality test asks a series of questions to rate an individual's likes and dislikes. The test will then classifies the personality type as either a blue, green, orange or gold with characteristic and colour specific traits. The subject may be a combination of two colours, but usually he/she will exhibit one primary colour. This personality test is not a means to type cast an individual but to understand the personality attributes associated with various people. The subject generally will operate as one primary colour for the most part of his existence, but high levels of stress and other environmental factors can shift your personality type for short intervals. 
Ray Achievement Motivation Scale: A questionnaire of 14 items (3 point scale) was selected as the model questionnaire based on Ray-Lynn (An American Psychologist and Researcher) who worked on achievement motivation and his master piece in the field of research is development and validation of achievement motivation scale. It was modified by JJ Ray, an Australian psychologist who prepared this questionnaire for a quick evaluation for Achievement Motivation. It was used in order to measure the variable of achievement motivation of the sample.

Statistical Analyses: The data collected was analysed and processed using SPSS(Statistical Package for Social Science) Software. 'T-Test' (Level of Significant 5\%) was performed for analysing and understanding the achievement motivation of the subjects. 'One Way Anova' (Level of Significant 5\%) was used for the study and classification of personality types into 4 colours: Orange, Green, Blue and Gold. The correlation between these two parameters through Pearson's Correlation Coefficient was tabulated from the investigation.

\section{RESULT AND DISCUSSION}

The analysis of the data collected from the considered sample yielded the following results:

\section{Table-1:}

H1: There is no significant difference in the level of Achievement Motivation between male and female.

\begin{tabular}{|l|l|l|l|l|l|}
\hline Gender & $\mathbf{N}$ & Mean & SD & 't' value & Significance \\
\hline M & 57 & 32.19 & 5.255 & \multirow{2}{*}{$0.42^{\mathrm{NS}}$} & \multirow{2}{*}{1.96} \\
\hline F & 53 & 31.65 & 3.256 & & \\
\hline
\end{tabular}

The table- 1 shows Mean, Standard deviation and the ' $t$ ' value of Achievement Motivation among male and female. The mean and standard deviation score on Achievement Motivation of male are 32.19 and 5.255 respectively. The mean and standard deviation score on Achievement Motivation of female are 31.65 and 3.256 respectively. The ' $t$ ' value (0.42) indicates that there is no significant difference is found between the two groups in the level of Achievement Motivation. Hence hypothesis is accepted. Hence it is evident that an individual cannot be tagged as ambitious just on the mere basis of gender. The motivation for achieving and succeeding solely depends upon an individual's interests, upbringing, nature and personality. Both male and female have the sense of competence and compete each other in different fields. Now a days the gender discrimination is almost absent in many field. Both of them have their clear vision about their goals. Through the knowledge in technology, media and social exposure they know the ways to achieve their goals. 
Table-2:

H2: There is no significant difference on the level of Achievement Motivation on the basis of Colour based Personality.

\begin{tabular}{|l|l|l|l|}
\hline Colour & Mean & 'F' value & Significance \\
\hline Orange & \multirow{2}{*}{24.719} & & \\
\cline { 1 - 2 } Green & 34.795 & \multirow{2}{*}{$3.92^{*}$} & \multirow{2}{*}{3.68} \\
\cline { 1 - 2 } Blue & 33.871 & & \\
\cline { 1 - 2 } Gold & 37.160 & & \\
\hline
\end{tabular}

Table-2 shows that level of the Achievement motivation among the Colour based Personality. The F-value shows that there is a significant difference in the level of Achievement Motivation on the basis of Colour based Personality of the subjects. Hence hypothesis is not accepted, i.e., there is a significant difference in these four types of personality classified on the basis of the colours Orange, Green, Blue and Gold. Hence, each individual has his own characteristic traits which may be either innate or developed and acquired through experiences during the lifetime. The personality traits are more responsible for the individual behaviour, attitude, and their value. The personality traits will make an individual in a way to adjust with the environment. The differences in the colour based personality will automatically shows the difference in person's behaviour patterns, cognition, emotions, perception and acquired values as well as life experiences.

\section{Table-3}

H3: There is no relationship between Achievement Motivation and Colour based Personality.

\begin{tabular}{|l|l|l|}
\hline Sl. No. & Colour & Achievement Motivation \\
\hline 1. & Orange & 0.38 \\
\hline 2. & Blue & 0.285 \\
\hline 3. & Green & $0.432^{*}$ \\
\hline 4. & Gold & -0.286 \\
\hline
\end{tabular}

The following conclusions can be drawn from the Pearson's correlation coefficient to establish a relationship between Achievement Motivation and Colour based Personality types. The table shows that the Colour based Personality of Orange having the predominant characteristics of being Spontaneous, Impulsive, Generous, Optimistic, Eager, Bold, Immediate and Courageous and the Personality of Blue having the Characteristics of Enthusiastic, Sympathetic, Idealistic, Spiritual, Sincere, Peaceful, Flexible and Imaginative are positively correlate with the level of 
Achievement Motivation but that is not in the significant level. Whereas the Colour based Personality of Green is significantly positively correlated with Achievement Motivation

Personality types based on the colour Green significantly positively correlate with the Achievement Motivation. These subjects show common traits of being analytic, logical, conceptual, abstract and inventive. These characteristics are very essential for boosting an individual's self-belief and thus enhance the level of Achievement Motivation.

Individuals who are grouped under the colour Gold have a negative Pearson's Correlation coefficient indicating that the two parameters considered are inversely related. It is possible that due to the traits like lack of order, Dependable, Sensible and Anxious.

\section{CONCLUSION}

The survey was conducted among 110 college students of age group 18-22 years to assess the existing relationship between the level of Achievement Motivation and the Colour Personality. The Collected data were analysed by using SPSS package. It was concluded that in the level of Achievement Motivation there is no difference between male and female. Personality types based on the colour Green preference was positively correlated with the Achievement Motivation and these individuals were more logical, analytical, abstract. Personality types based on the colour Gold preference have significantly negative correlation who lack order, were less Dependable, and Anxious.

\section{BIBLIOGRAPHY}

Aihua Tao, Xing Li, “Achievement Motivation Improving and its Relationship with Personality Based on the Teaching Reform of Psychology,” International Journal of Education and Research Vol. 2 No. 8 August 2014.

Divya Ghorawat, Ravina Madan, "Correlation between Personality Type and Color Shade Preference,” The International Journal of Indian Psychology: Volume: 01 | Issue: 04 | ISSN 2348-5396.

Rense Lange,Jason Rentfrow, “Color and Personality: Strong’s Interest Inventory and Cattell's 16PF, ” North American Journal of Psychology Vol.9 No.3 December 2007.

Ekta Sharma, “Personality Mapping: Tool to Analyze Achievement Orientation,’ iBusiness, 2013, 5, 59-64. Published Online June 2013 (http://dx.doi.org/10.4236/ib.2013.52007)

Sharma S, "A Study on Effect of Colors on Personality and its Relation with Spirituality," Research Journal of Family, Community and Consumer Sciences _ ISSN 2320 - 902X Vol. 3(1), 1-3, March (2015).

RESEARCH ARTICLE UDC 159.937.51 (Dunja Cigić, Vojislava Bugarski )

Webs

http://jonjayray.tripod.com/quickao.html (JJ Ray,1979)

http:/www.goodreads.com/topic/show/1092168-what-your-favourite-color-says-about-yourpersonality

http://psychologia.co/personality-color/

http://www.empower-yourself-with-color-psychology.com/personality-color.html 